\title{
Load model and modified model medical stone on the removal of arsenic in drinking water
}

\author{
Gang Zhang, Meng Zhao \& Fei Yang \\ Kumming University of Science and Technology, Kunming 650504, China
}

\begin{abstract}
Using the method which potassium permanganate modified medical stone and titanium dioxide load maifanite to removal arsenic in drinking water. Compared with the dosage, shaking time, $\mathrm{pH}$ on arsenic removal, the research results show that when the dosage is $0.2 \mathrm{~g}$, the effect of titanium dioxide and potassium permanganate load maifanite modified medical stoneare are better; when the time was 40min, the shock load Maifanites titanium dioxide removal rate of arsenic reach the health standards of drinking water, while the high potassium permanganate modified maifanite shock about $60 \mathrm{~min}$,which meet the requirements; when $\mathrm{pH}=6, \mathrm{KMnO}_{4}$ modified maifanite up to the standards, while the load of stone in the titanium dioxide is between $\mathrm{pH}=5-7$ can meet adsorption performance requirements and its $\mathrm{pH}$ had little effect. According to the comprehensive comparison, using titanium dioxide load maifanite is better than potassium permanganate modified Maifanshiarsenic in dealing with the effects of arsenic in drinking water.
\end{abstract}

KEYWORD: Modified medical stone, Load medical stone, Arsenic, Adsorb, Contrast

\section{INTRODUCTION}

With the continuous development of China's heavy industry, the heavy metal pollution of groundwater in China has become more and more serious, especially arsenic pollution [1].Arsenic mainly exists in the form of compounds in the water, and arsenic compounds are the main source of water pollution, and pure metal arsenic will not cause pollution. Traditional arsenic removal process is mainly chemical precipitation method [2-4], its efficiency is low, the cost is high, and some still exist secondary pollution problems [5-7]. Therefore, sewage treatment subject to certain restrictions, and it is necessary to seek an efficient and economical adsorbent to replace.

The medical stone itself has a porous and spongy structure [8-9], it's specific surface area is very large, and its interior contains abundant adsorbed minerals, but if using single medical stone to remove arsenic, the effect not very ideal; Size of the nano titanium dioxide [10] itself belongs to the nanometer level, is very small, so its surface energy is also higher. At the same time, the experiment found that a single nanometer titanium dioxide on the adsorption effect of arsenic in water is very significant, but the price is expensive.

\section{EXPERIMENTAL PART}

\subsection{Reagents and instruments.}

Materials: Experimental water sample is raw water sample simulated that was simulated by distilled water; Preparation of arsenic standard solution (1000 $\mathrm{g} / \mathrm{mL}$, national non ferrous metal and electronic material analysis test center); The Chinese medical stone (Inner Mongolia Pingdingshan Naiman Banner in Tongliao City); Nano titanium dioxide, purity of 99.8\%, particle size 5-10nm, anatase, National Pharmaceutical Group Chemical Reagent Co., ltd..

Instruments: phs-3c pH meter, electronic balance, $722 \mathrm{~N}$ type spectrophotometer, oven, muffle furnace, low speed centrifuge (Shanghai Anting Scientific Instrument Factory), the HY-4 adjustable speed multi use oscillator (Jintan City, Jiangsu Province, the earth Automatic Instrument Factory), the ultrasonic oscillator.

\section{$2.2 \mathrm{KMnO}_{4}$ modified medical stone preparation.}

First washing the Chinese medical stone that particle size was $5 \mathrm{~mm}$ with deionized water repeatedly, and put it in the oven under thecondition of $105^{\circ} \mathrm{C}$ and dry for $24 \mathrm{~h}$, after drying theseal placed in the shade to stay prepared. Then taking the ordinary beaker 
in the laboratory, add $100 \mathrm{ml} 0.1 \mathrm{~mol} / \mathrm{L} \mathrm{KMnO}_{4}$ solution, put the beaker at HY-4 multi speed oscillator of $25^{\circ} \mathrm{C}$ and shock for 30min. Finally removing the medical stone into the constant temperature drying box, drying and sealing.

\subsection{Load medical stone of $\mathrm{TiO}_{2}$ preparation.}

Take the ordinary laboratory beaker, add $100 \mathrm{ml}$ deionized water and $1.0 \mathrm{~g}$ nano $\mathrm{TiO} 2$ powder, and stir well. The nanometer titanium dioxide has the characteristics of easy precipitation, so it is necessary to add a certain amount of K12 to the beaker, which can make nano titanium dioxide suspended in the beaker uniformly for $30 \mathrm{~min}$. Then put the beaker in an ultrasonic oscillator, so that the nano carbon dioxide can be uniformly dispersed in the deionized water. Finally add a certain amount of medical stone that has been cleaned to the beaker, then stir it for 30 min with a glass rod, remove the medical stone, and wash it repeatedly with deionized water, then place it in the constant temperature drying box of $105^{\circ} \mathrm{C}$ for drying, and sealing preservation reserve.

\section{CONCLUSION AND ANALYSIS}

The effects of the three aspects of dosage, oscillation time and $\mathrm{pH}$ on the removal efficiency of arsenic in drinking water were compared. The reason to consider the three aspects, mainly because in the actual production and life, economic factors are the important factors that restrict the development of the new technology and the process of the new technology. Consider these three factors in the actual operation of the process, which can save the cost of investment and reduce the cost of the operation of the process. It has a reference value for the removal of heavy metal arsenic in drinking water.

\subsection{Effect of dosage on the removal rate of arsenic.}

Select 12 specifications for the volumetric flask of $250 \mathrm{ml}$, divide it into two groups, A and B, and numbers $(1,2,3,4,5,6)$. Then adding modified medical stone by $\mathrm{KMnO}_{4}(0.1 \mathrm{~mol} / \mathrm{L})$ to $\mathrm{A}$ group, and adding load medical stone with nano titanium dioxide to the B group, respectively, 0.01, 0.02, 0.05, 0.10, 0.15, $0.2 \mathrm{~g}$. At the same time, $100 \mathrm{~mL}$ arsenic solution with initial concentration of $100 \mathrm{mg} / \mathrm{L}$ were added to A and $\mathrm{B}$ group respectively, and $\mathrm{pH}$ value in water samples was adjusted to 7 by the HC1 solution with the concentration of $0.5 \mathrm{~mol} / \mathrm{L}$ and the $\mathrm{NaOH}$ solution with the concentration of $1.0 \mathrm{~mol} / \mathrm{L}$. Then continue to shake it with $160 \mathrm{r} / \mathrm{min}$ rate in the 25 degrees thermostatic shaker, shake and absorption for $60 \mathrm{~min}$. Finally take the supernatant liquid after a period of time till the conical flask is stationary, and test the content of arsenic in the solution. The experimental results are shown in figure 1.

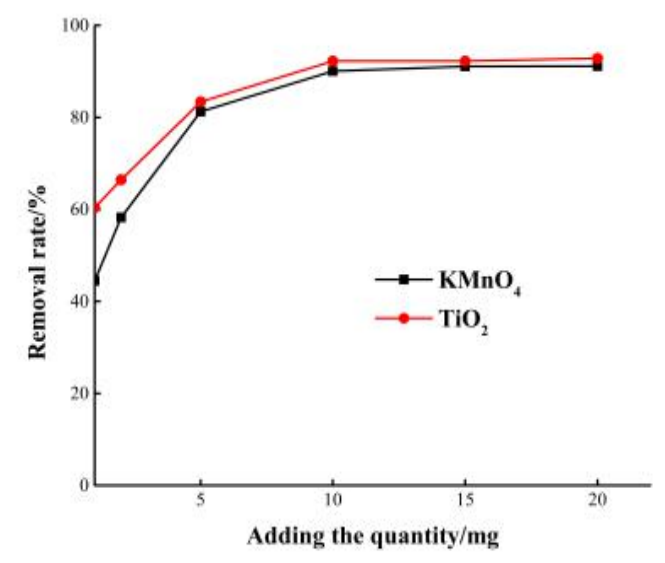

Fig.1 Effect of dosage on removal efficiency of arsenic

From Figure 1, it can be seen, with the increase of the dosage of load medical stone with nano titanium dioxide and modified medical stone by $\mathrm{KMnO}_{4}$, the removal efficiency of arsenic is getting better and better. It is because that there is a wealth of porosity within the medical stone, and nano titanium dioxide itself with high specific surface area. At the same time, the nanometer titanium dioxide load on the medical stone can further increase the surface energy of nanometer titanium dioxide, so the removal efficiency of arsenic is considerable. When the dosage arrive at $1.0 \mathrm{~g}$, the removal rate of arsenic of load medical stone with nano titanium dioxide reached $92.24 \%$, while the modified medical stone by $\mathrm{KMnO}_{4}$ only reached $90.06 \%$. There is no obvious effect on the removal efficiency by adding the two kinds of medical stone constantly. Therefore, by comparing of the removal rate of arsenic of load medical stone with nano titanium dioxide and modified medical stone by $\mathrm{KMnO}_{4}$, and its comprehensive application in practical application, it is not difficult to find that when nanometer titanium dioxide load stone as the carrier of arsenic adsorption, the dosage selection is appropriate for the $1.0 \mathrm{~g}$.

\subsection{Effect of oscillation time on the removal rate of arsenic.}

Select 16 specifications for the volumetric flask of $250 \mathrm{ml}$, divide it into two groups, A and B, and numbers $(1,2,3,4,5,6,7,8)$. Then adding modified medical stone by $\mathrm{KMnO}_{4}(0.1 \mathrm{~mol} / \mathrm{L})$ of $0.20 \mathrm{~g}$ to $\mathrm{A}$ group, and adding load medical stone with nano titanium dioxide of $0.20 \mathrm{~g}$ to the $\mathrm{B}$ group. At the same time, $100 \mathrm{~mL}$ arsenic solution with initial concentration of $100 \mathrm{mg} / \mathrm{L}$ were added to A and B group respectively, and the $\mathrm{pH}$ value of the water sample is adjusted to 7 . Then put these volumetric flask on the HY-4 adjustable speed multi use oscillator to shake and absorption for 10, 20, 30, 40, 50, 60, 70, 80min 
respectively. Finally take the supernatant liquid after a period of time till the conical flask is stationary, and test the content of arsenic in the solution. The experimental results are shown in figure 2.

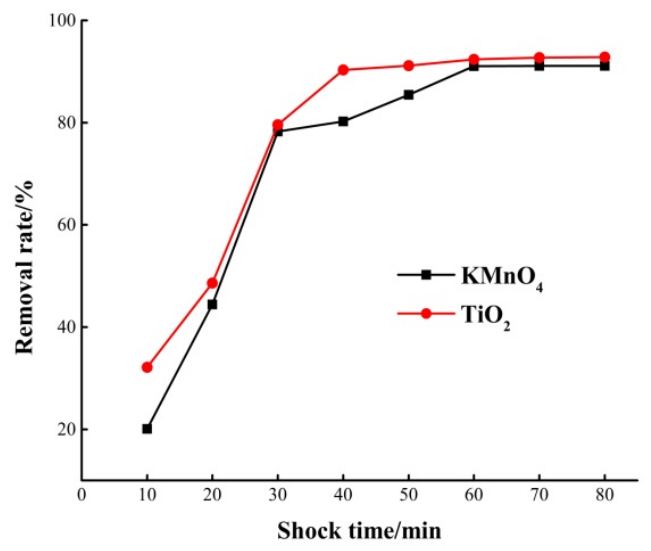

Fig.2 Effect of oscillation time on the removal rate of arsenic

As can be seen from figure two, with the continuous extension of the shock time, the effect of the two lines on the removal of arsenic gradually became stronger. When the shock time reached $60 \mathrm{~min}$, the adsorption gradually stabilized. When the oscillation time is $60 \mathrm{~min}$, the removal rate of arsenic of load medical stone with nano titanium dioxide reached $92.37 \%$, while the modified medical stone by $\mathrm{KMnO}_{4}$ only reached $91.06 \%$. There is no obvious effect on the removal efficiency by increasing the time of oscillation constantly. Therefore, by comparing of the removal rate of arsenic of load medical stone with nano titanium dioxide and modified medical stone by $\mathrm{KMnO}$, and its comprehensive application in practical application, it is not difficult to find that when nanometer titanium dioxide load stone as the carrier of arsenic adsorption, the oscillation time is appropriate for $60 \mathrm{~min}$.

\subsection{Effect of $p H$ value on the removal rate of arsenic.}

Select 16 specifications for the volumetric flask of $250 \mathrm{ml}$, divide it into two groups, A and B , and numbers $(1,2,3,4,5,6,7,8)$.Then adding modified medical stone by $\mathrm{KMnO}_{4}(0.1 \mathrm{~mol} / \mathrm{L})$ of $0.20 \mathrm{~g}$ to A group, and adding load medical stone with nano titanium dioxide of $0.20 \mathrm{~g}$ to the $\mathrm{B}$ group. At the same time, $100 \mathrm{~mL}$ arsenic solution with initial concentration of $100 \mathrm{mg} / \mathrm{L}$ were added to $\mathrm{A}$ and $\mathrm{B}$ group respectively. Then the $\mathrm{pH}$ values of the two groups of water samples were adjusted to 2, 3, 4, 5, 6, 7, 8, 9, respectively, and put these volumetric flask on the HY-4 adjustable speed multi use oscillator to shake and absorption for $60 \mathrm{~min}$. Finally take the supernatant liquid after a period of time till the conical flask is stationary, and test the content of arsenic in the solution. The experimental results are shown in figure 3.

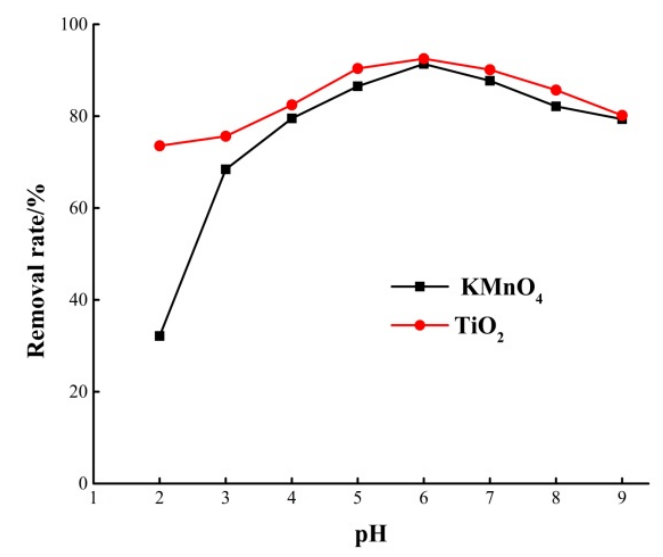

Fig.3 Effect of $\mathrm{pH}$ value on the removal rate of arsenic

As can be seen from the figure 3 , with the increase of $\mathrm{pH}$ in the solution at the beginning of the reaction phase of the two groups of samples, the corresponding to the adsorption efficiency of arsenic gradually strengthened. The reaction adsorption efficiency of modified medical stone by $\mathrm{KMnO}_{4}$ is low at the beginning stage, this is due to the smaller $\mathrm{pH}$ resulting in the surface layer or structure of medical stone dissolved a lot of aluminosilicate and affected the adsorption sites of $\mathrm{MnO}_{2}$. But the effect of $\mathrm{pH}$ on load medical stone with nano titanium dioxide is not very significant, the adsorption activity of titanium dioxide in acidic or alkaline conditions can still maintain a stable state. The the removal rate of arsenic of modified medical stone by $\mathrm{KMnO}_{4}$ can reach drinking water health indicators when $\mathrm{pH}=6$, but the removal rate of arsenic of load medical stone with nano titanium dioxide can meet the drinking water health indicators when $\mathrm{pH}=5-7$. Therefore, the $\mathrm{pH}=6$ is appropriate when nanometer titanium dioxide load stone as the carrier of arsenic adsorption.

\section{SUMMARY}

In view of the phenomenon of excessive arsenic in drinking water, this paper adopts two methods to modify medical stone, and analyze the removal rate of arsenic by means of comparison. The conclusion shows that the effects are great of load medical stone with nano titanium dioxide and modified medical stone by $\mathrm{KMnO}_{4}$ when the dosage is $0.2 \mathrm{~g}$; When the concussion time is $40 \mathrm{~min}$, load medical stone with nano titanium dioxide can reach drinking water health indicators, but modified medical stone by $\mathrm{KMnO}_{4}$ only can meet the standard when the concussion time is 60min; The modified medical stone by $\mathrm{KMnO}_{4}$ up to standard when the $\mathrm{pH}=6$, but load medical stone with nano titanium dioxide between $\mathrm{pH}=5-7$ can meet the requirements. Meanwhile, $\mathrm{pH}$ has little effect on its adsorption properties. After 
comprehensive comparison, it can be seen that load medical stone with nano titanium dioxide is better than modified medical stone by $\mathrm{KMnO}_{4}$ for the removal of arsenic in drinking water. Therefore, it is better to select load medical stone with nano titanium dioxide to remove arsenic in drinking water.

\section{REFERENCES}

[1] Jin Yinlong, Liang Chaoju, He Gongli. China endemic arsenic poisoning distribution investigation (General report), J. Health research. 32(6), (2003)519-539.

[2] Zhuang Minglong, Chai Liyuan. Arsenic wastewater treatment is reviewed. Industrial water treatment.5 (2), (2004)1316.

[3] Wang Ying, Lv Sidan, Li Xin. The research progress of removal of arsenic in water and prospect,J. Environmental science and technology.33(9),(2010)102-107.

[4] He Jingjing. Modified activated carbon removal in the raw water research of antimony. Kunming university of science and technology, 2014.

[5] Huang Xin, Gao Naiyun, Liu Cheng. Drinking water arsenic removal technology are reviewed, J.Water purification technology.26(s), (2007)40-44.

[6] Liang Meina, Zhu Yinian, Zhu Fengqi. The adsorption effect of manganese dioxide As (v) in water research, J. Environmental protection science and technology. 14(2), (2008) 27-31.

[7] Ma Lin, Tu Shuxin. Water arsenic removal material screening and its chemical composition characteristics research, J. Water treatment technology.37 (1), (2011) 68-72.

[8] Li Juan, Zhang Panyue. Physico-chemical properties of the medical stone and its application in water quality, J. Environmental science and technology.31 (10), (2008) 63-75.

[9] Chen Li, Li Feng. Determination of the Chinese medical stone surface properties and adsorption mechanism of the research, J. Inner Mongolia petrochemical industry. 4(24), (1998)18-20.

[10] Wang Shuqin, Song Limin. Study of nanometer titanium dioxide arsenic wastewater treatment, J. Industrial safety and environmental protection.33 (7), (2007) 14-16. 